\title{
Sistema de partículas no grafo completo com remoção ao pular
}

\author{
Mario Andres Estradall \\ CCEN-Universidade Federal de Pernambuco, Recife, PE
}

\begin{abstract}
Resumo.
Estudamos um sistema de partículas no grafo completo, no qual cada partícula é retirada após visitar um vértice e/ou acordar uma partícula dormente se o vértice contiver uma. Esta é uma variação do modelo conhecido como modelo dos sapos com tempo de vida não geométrico. Consideramos que o processo começa com uma partícula ativa em um único vértice. Mostramos que a proporção de vértices visitados e que o tempo de absorção do processo convergem em probabilidade para zero quando a quantidade de vértices no grafo completo tende para infinito.
\end{abstract}

Palavras-chave. Modelo dos sapos, grafo completo, passeios aleatórios, modelo não markoviano.

\section{Introdução}

O modelo dos sapos é um modelo estocástico para a propagação de uma epidemia em um grafo, no qual uma partícula dormente passa a realizar um passeio aleatório simples e a acordar outras partículas, uma vez que se torna ativa. Estudamos uma versão do modelo dos sapos no grafo completo com $N+1$ vértices, em que cada partícula ativa é removida ao pular e se o vértice escolhido por esta partícula tiver uma partícula dormente, ela é acordada e começa a realizar um passeio aleatório simples na estrutura geométrica, independente da trajetória realizada pela partícula anterior. Por simplicidade nos referiremos a este modelo pelo nome Pula-Morre. Este modelo foi introduzido pela primeira vez em $\mathbb{Z}$ por Lebensztayn et al. [7, os autores consideraram que cada partícula ativa pode realizar $L$ saltos para vértices escolhidos uniformemente ao acaso, ativando todas as partículas inativas que encontra ao longo do seu caminho. Se $L=1$, o modelo dos autores coincide com nosso modelo. Estudamos o tempo em que todas as partículas se tornam inativas, denotado de tempo de absorção, como também a proporção de vértices visitados ao finalizar o grafo.

Os tempos aleatórios têm sido um dos focos no estudo do modelo dos sapos em diferentes estruturas geométricas e considerando diferentes tempos de vida das partículas. A variável aleatória tempo de acordar, é entendida como o tempo que lhe tomam a todas as partículas acordarem no grafo completo, ela tem sido amplamente estudada em Frieze and Grimmett [3, Doerr and Künnemann 2 e Pittel 8]. Em todas estas, considera-se que existe uma partícula em cada um dos vértices do grafo completo e como condição inicial considera-se que uma delas está ativa e as outras inativas. Se considera que as partículas ativas não são removidas em nenhum instante de tempo. Uma partícula se move seguindo a dinâmica de um passeio aleatório simples, ativando as demais ao visitar um vértice contendo uma partícula inativa. Os autores em Cartern et al. [1, fizeram um resumo dos resultados abordando este tema, além mostraram uma prova para o valor esperado do tempo de acordar ser $\Theta(\log n)$, em que $n$ a quantidade de vértices no grafo completo.

\footnotetext{
${ }^{1}$ marioestradalopez@gmail.com, mael1@de.ufpe.br
} 
Neste trabalho mostramos que o tempo de absorção para o processo denominado Pula-Morre é de $o_{p}(N+1)$, entendendo $X_{n}=o_{p}\left(b_{n}\right)$ se para todo número real $\varepsilon>0$ e para todo número real $\eta>0$ existir um número inteiro positivo $n_{0}=n_{0}(\varepsilon, \eta)$, tal que

$$
\mathbb{P}\left(\left|X_{n} / b_{n}\right| \geq \varepsilon\right)<\eta, \quad \forall n \geq n_{0} .
$$

\section{Representação estocástica}

O processo consiste em duas ações principais, pular e ocupar. Elas podem ser pensadas como operações que acontecem em instantes alternativos entre $t$ e $t+1$ mas só são registradas no tempo $t+1$, para qualquer instante de tempo. Assuma-se um grafo completo com $N+1$ vértices denotado por $K_{N+1}$ e suponha que há dois tipos de partículas: partículas ativas e inativas. Como condição inicial assumimos que um vértice em $K_{N+1}$ tem uma partícula ativa e as outras partículas estão inativas. Denota-se o número de partículas ativas no tempo $t$ como $A_{t}$ e o número de vértices não visitados como $I_{t}$. O número de vértices visitados no tempo $t$ é denotado por $V_{t}=N+1-I_{t} \mathrm{e}$ o número de partículas mortas até o instante $t$ é $D_{t}=V_{t}-A_{t}$. Conhecido o estado do processo $\left(I_{t}, A_{t}, D_{t}\right)$ no tempo $t$, consideram-se duas variáveis aleatórias auxiliares, denotadas por $X_{t+1} \mathrm{e}$ $Z_{t+1}$.

Como foi dito na Introdução, consideramos uma única partícula ativa mas é possível também considerar $k$ partículas ativas na condição inicial. A seguinte descrição considera $k \geq 1$, as partículas ativas pulam com probabilidade 1, tentando atingir um vértice não visitado, devido que no caso contrário o processo finalizaria. A dinâmica anteriormente citada pode ser descrita, no tempo $t+1$, por: $Z_{t+1} \sim \operatorname{Binomial}\left(A_{t} ; \frac{I_{t}}{N}\right)$. A geração de novas partículas vem determinada pelos vértices inativos que são visitados pelas partículas ativas. Para conhecer a quantidade de partículas novas no sistema no tempo $t+1$, ao número de vértices não visitados no tempo anterior lhe subtraímos o número de vértices inativos no tempo $t+1$. O número de vértices não visitados, no tempo $t+1$, vem especificado por: $I_{t+1} \sim \operatorname{EmpBox}\left(Z_{t+1} ; I_{t}\right)$, para mais informação sobre EmpBox, veja-se Apêndice A. Descrevemos o processo como segue:

$$
\left\{\begin{array}{l}
I_{t+1}=I_{t}-Y_{t+1} . \\
A_{t+1}=Y_{t+1} \mathrm{e} \\
D_{t+1}=V_{t+1}-A_{t+1}=N+1-I_{t+1}-A_{t+1}=N+1-I_{t} .
\end{array}\right.
$$

Onde $Y_{t+1}$ é a variável aleatória que representa o número de vértices ocupados no tempo $t+1$.

\section{Resultados principais}

\subsection{Proporção de vértices visitados}

Seja $\mathcal{G}_{t}=\sigma\left(\left\{\left(I_{u}, A_{u}, D_{u}\right), 0 \leq u \leq t\right\}\right)$ a sigma álgebra gerada pelo vetor aleatório até o tempo $t$, representando a informação acumulada depois de $t$ passos do modelo dos sapos com o segundo mecanismo de remoção. Redefinimos neste capítulo o valor esperado, a variância e a covariância condicionada à filtração $\mathcal{G}_{t} \operatorname{como:~} \mathbb{E}(\cdot)=E\left(\cdot \mid \mathcal{G}_{t}\right)$ e $\mathbb{V}(\cdot)=\operatorname{Var}\left(\cdot \mid \mathcal{G}_{t}\right)$.

Lemma 3.1. As expressões para os valores esperados condicionados a $\mathcal{G}_{t}$ para $I_{t+1}, A_{t+1}$ e $D_{t+1}$ 
são:

$$
\begin{aligned}
\mathbb{E}\left(I_{t+1}\right) & =I_{t}\left(1-\frac{1}{N}\right)^{A_{t}}, \\
\mathbb{E}\left(A_{t+1}\right) & =I_{t}\left[1-\left(1-\frac{1}{N}\right)^{A_{t}}\right], \\
\mathbb{E}\left(D_{t+1}\right) & =N+1-I_{t},
\end{aligned}
$$

respectivamente.

Demonstração. Usando a expressão (1) no Apêndice A, calculamos:

$$
\begin{aligned}
\mathbb{E}\left(I_{t+1} \mid Z_{t+1}\right) & =E\left(I_{t+1} \mid Z_{t+1}, \mathcal{G}_{t}\right) \\
& =I_{t}\left(1-\frac{1}{I_{t}}\right)^{Z_{t+1}}
\end{aligned}
$$

O que implica, tomando novamente valor esperado, que

$$
\begin{aligned}
\mathbb{E}\left(I_{t+1}\right) & =E\left(E\left(I_{t+1} \mid Z_{t+1}, \mathcal{G}_{t}\right) \mid \mathcal{G}_{t}\right) \\
& =E\left(I_{t}\left(1-\frac{1}{I_{t}}\right)^{Z_{t+1}} \mid \mathcal{G}_{t}\right) \\
& =I_{t}\left(1-\frac{I_{t}}{N}\left(1-1+\frac{1}{I_{t}}\right)\right)^{A_{t}} \\
& =I_{t}\left(1-\frac{1}{N}\right)^{A_{t}} .
\end{aligned}
$$

- Valor esperado $A_{t+1}$ :

$$
\begin{aligned}
\mathbb{E}\left(A_{t+1}\right) & =\mathbb{E}\left(I_{t}\right)-\mathbb{E}\left(I_{t+1}\right), \\
& =I_{t}-I_{t}\left(1-\frac{1}{N}\right)^{A_{t}} \\
& =I_{t}\left[1-\left(1-\frac{1}{N}\right)^{A_{t}}\right] .
\end{aligned}
$$

- Valor esperado $D_{t+1}$ :

$$
\mathbb{E}\left(D_{t+1}\right)=N+1-I_{t} .
$$

Usando o Lema 3.1 e os momentos no Apêndice A temos que

Lemma 3.2. As expressões para a variância condicionada a $\mathcal{G}_{t}$ de $I_{t+1}, D_{t+1}$ e $A_{t+1}$ são:

$$
\begin{aligned}
\mathbb{V}\left(I_{t+1}\right) & =I_{t}\left\{\left(I_{t}-1\right)\left(1-\frac{2}{N}\right)^{A_{t}}-I_{t}\left(1-\frac{1}{N}\right)^{2 A_{t}}+\left(1-\frac{1}{N}\right)^{A_{t}}\right\}, \\
\mathbb{V}\left(D_{t+1}\right) & =0 e \\
\mathbb{V}\left(A_{t+1}\right) & =\mathbb{V}\left(I_{t+1}\right),
\end{aligned}
$$

respectivamente. 
Demonstração. Tendo como objetivo aplicar a fórmula da decomposição da variância, calculem-se

- Variância de $I_{t+1}$ : Temos que $\mathbb{E}\left(I_{t+1} \mid Z_{t+1}\right)=I_{t}\left(\frac{I_{t}-1}{I_{t}}\right)^{Z_{t+1}}$ e por 2 do Apêndice A. temos que $\operatorname{Var}\left(I_{t+1} \mid Z_{t+1}, \mathcal{G}_{t}\right)=I_{t}\left(I_{t}-1\right)\left(\frac{I_{t}-2}{I_{t}}\right)^{Z_{t+1}}+I_{t}\left(\frac{I_{t}-1}{I_{t}}\right)^{Z_{t+1}}-I_{t}^{2}\left(\frac{I_{t}-1}{I_{t}}\right)^{2 Z_{t+1}}$, em consequência podemos concluir que:

$$
\begin{aligned}
\mathbb{V}\left(I_{t+1}\right)= & I_{t}\left(I_{t}-1\right)\left(1-\frac{2}{N}\right)^{A_{t}}+I_{t}\left(1-\frac{1}{N}\right)^{A_{t}}-I_{t}^{2} E\left(\left(\frac{I_{t}-1}{I_{t}}\right)^{2 Z_{t+1}} \mid \mathcal{G}_{t}\right)+ \\
& +I_{t}^{2} E\left(\left(\frac{I_{t}-1}{I_{t}}\right)^{2 Z_{t+1}} \mid \mathcal{G}_{t}\right)-I_{t}^{2}\left(1-\frac{1}{N}\right)^{2 A_{t}}, \\
= & I_{t}\left(I_{t}-1\right)\left(1-\frac{2}{N}\right)^{A_{t}}+I_{t}\left(1-\frac{1}{N}\right)^{A_{t}}-I_{t}^{2}\left(1-\frac{1}{N}\right)^{2 A_{t}} .
\end{aligned}
$$

- Variância de $D_{t+1}$ e $A_{t+1}$ :

$$
\begin{aligned}
\mathbb{V}\left(D_{t+1}\right) & =\mathbb{V}\left(N+1-I_{t}\right), \\
& =0 .
\end{aligned}
$$

Por outro lado, temos que $A_{t+1}=Y_{t+1}=I_{t}-I_{t+1}$, em consequência temos que: $\mathbb{V}\left(A_{t+1}\right)=$ $\mathbb{V}\left(I_{t+1}\right)$.

Consideramos o seguinte sistema dinâmico a tempo discreto, $\varrho_{t}^{(N)}=\left(\tilde{\iota}_{t}^{(N)}, \tilde{\alpha}_{t}^{(N)}, \tilde{\delta}_{t}^{(N)}\right)$, em que

$$
\left\{\begin{array}{l}
\tilde{\iota}_{t+1}=\tilde{\iota}_{t} e^{-\tilde{\alpha}_{t}}, \\
\tilde{\alpha}_{t+1}=\tilde{\iota}_{t}\left(1-e^{-\tilde{\alpha}_{t}}\right), \\
\tilde{\delta}_{t+1}=1-\tilde{\iota}_{t}, \\
\tilde{\iota}_{0}=\frac{N}{N+1}, \quad \tilde{\alpha}_{0}=\frac{1}{N+1}, \quad \tilde{\delta}_{0}=0 .
\end{array}\right.
$$

As técnicas usadas em Lebensztayn and Estrada [6] mostram que o sistema acima se aproxima em probabilidade a sua contraparte estocástica $\eta_{t}:=\left(I_{t}, A_{t}, D_{t}\right) /(N+1)$ a medida que $N$ vai para infinito para todo $t$. Isto é, $\eta_{t}-\varrho t \stackrel{p}{\rightarrow} 0$ para todo $t$ quando $N \rightarrow \infty$, onde $\stackrel{p}{\rightarrow}$ denota convergência em probabilidade.

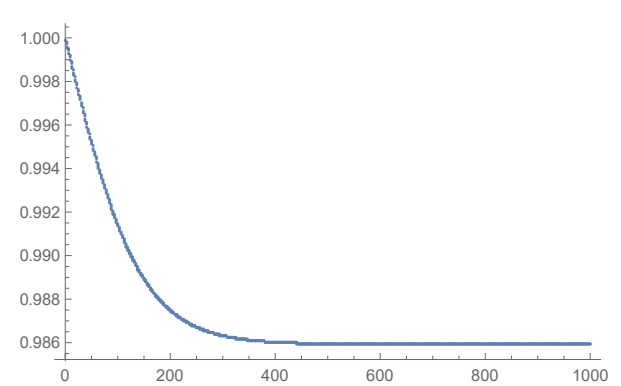

(a) $\tilde{\iota}_{t}$

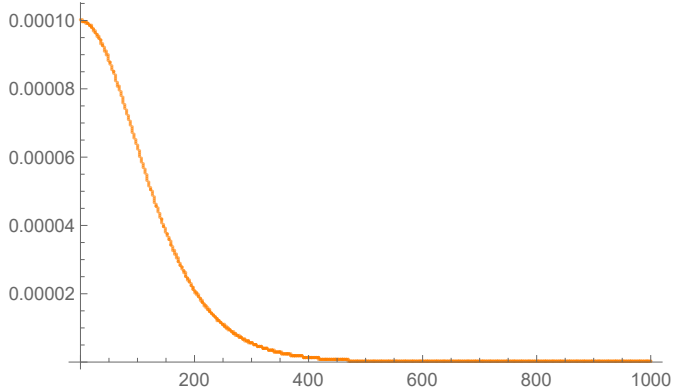

(b) $\tilde{\alpha}_{t}$

Figura 1: Gráficos para o sistema dinâmico $\varrho_{t}$ para $N=10000$. 
Observação 3.1. O sistema acima em (1) exibe uma única solução em $\tilde{\iota}_{\infty}=1$ quando $t \rightarrow \infty e$ $N \rightarrow \infty$. Podemos observar o anteriormente descrito na Figura 1 a, em que o sistema se estabiliza perto de um ponto aproximado a 1 para $N=10000$.

Defina $\lim _{t \rightarrow \infty} V_{t}=V_{\infty}$ e $\lim _{t \rightarrow \infty} I_{t}=I_{\infty}$. Devido que o sistema exibe uma única solução, $\tilde{\iota}_{\infty}=1$, concluímos que $\frac{I_{\infty}}{N+1} \stackrel{p}{\rightarrow} 1$ quando $N \rightarrow \infty$. Finalmente, já que $\frac{V_{t}}{N+1}=1-\frac{I_{t}}{N+1}$ para todo $t$, concluímos que $\frac{V_{\infty}}{N+1} \stackrel{p}{\rightarrow} 0$ quando $N \rightarrow \infty$.

O resultado anterior, também é valido considerando $k$ partículas em um dos vértices do grafo completo como condição inicial.

\subsection{Tempo de absorção}

A seguinte análise é considerando $k=1$. Defina o tempo de parada do processo, que é o tempo em que é absorvido o processo, como

$$
T=\min \left\{t: A_{t}=0\right\}
$$

Pelo Lema 3.1, temos que $\mathbb{E}\left(I_{t+1}\right)=I_{t}\left(1-\frac{1}{N}\right)$. Defina $m_{t}=I_{t-1}\left(1-\frac{1}{N}\right)^{-t+1}$, a expressão anterior é um martingal, isto é, $\mathbb{E}\left(m_{t}\right)=m_{t-1}$.

Consideremos a seguinte relação

$$
\begin{aligned}
P\left(\left|\left(\frac{N}{N-1}\right)^{T} \frac{I_{T}}{N+1}-\frac{I_{0}}{N+1}\right|>\varepsilon\right) & \leq \frac{\operatorname{Var}\left(\left(\frac{N}{N-1}\right)^{T} I_{T}\right)}{(N+1)^{2} \varepsilon^{2}} \\
& \leq \frac{\left(\frac{N}{N-1}\right)^{2 N} \operatorname{Var}\left(I_{T}\right)}{(N+1)^{2} \varepsilon^{2}}
\end{aligned}
$$

Aplicando a Lei da Variância Total, os Lema 3.1. Lema 3.2 e o Teorema da convergência dominada, obtemos que:

$$
\frac{\operatorname{Var}\left(I_{T}\right)}{(N+1)^{2}}=\frac{E\left(\mathbb{V}\left(I_{T}\right)\right)+\operatorname{Var}\left(\mathbb{E}\left(I_{T}\right)\right)}{(N+1)^{2}} \rightarrow 0 \quad \text { quando } N \rightarrow \infty
$$

Seja $f_{N}(x):=\left(\frac{N}{N-1}\right)^{x}(1-x)-\frac{N}{N+1}$. Notando que $I_{t}=A_{0}+I_{0}-T$, concluímos que

$$
\lim _{N \rightarrow \infty} \mathbb{P}\left(\frac{T}{N+1} \in\left(f_{N}^{-1}(-\varepsilon), f_{N}^{-1}(\varepsilon)\right)\right)=1 .
$$

Implicando

$$
\frac{T}{N+1} \stackrel{p}{\rightarrow} 0, \quad \text { quando } N \rightarrow \infty
$$

\section{Estudos de simulação}

Nesta Seção, ilustraremos por meio de estudos de simulação, o resultado obtido na Seção 3.2 . Na Figura 2 observamos 3000 realizações do tempo de absorção divido por $N+1$ representados pelos pontos azuis, em que o eixo $x$ representa o grau do grafo completo. Observamos que a medida que $N$ aumenta, as realizações vão se concentrando numa vizinhança de zero. As funções $f_{N}^{-1}(-\varepsilon)$ e $f_{N}^{-1}(\varepsilon)$ são representadas pelos pontos roxos, escolhendo $\varepsilon=\frac{1}{N}$. 


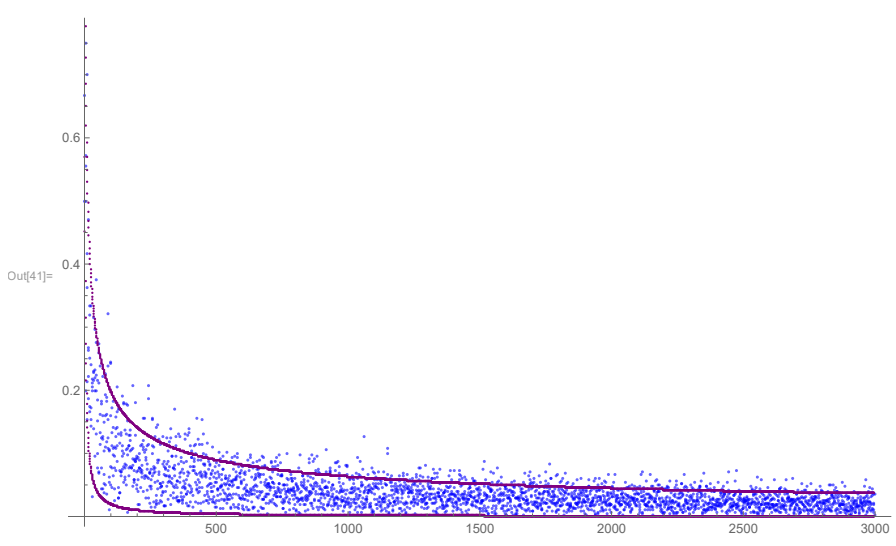

Figura 2: 3000 realizações para o tempo de absorção divido por $N+1$.

Na Figura 3 observamos 3000 realizações para o tempo de absorção representadas pelos pontos da cor magenta. Nessa figura, são representadas três funções $N^{2 / 3}, \sqrt{N}$ e $N^{1 / 3}$. A Figura 3 sugere que existe uma relação entre $T$ e $\sqrt{N}$. O autor em Harris [4] trabalhou com um mapeamento aleatório, cuja descrição coincide com o modelo Pula-Morre com uma partícula ativa, as abordagens usadas no artigo mostraram que o mapeamento com $m$ imagens sucessivas distintas tem valor esperado assintótico $\frac{1}{4} \sqrt{2 \pi N}$. O anterior, nos motiva a continuar trabalhando numa caraterização do tempo de absorção para $k>1$.

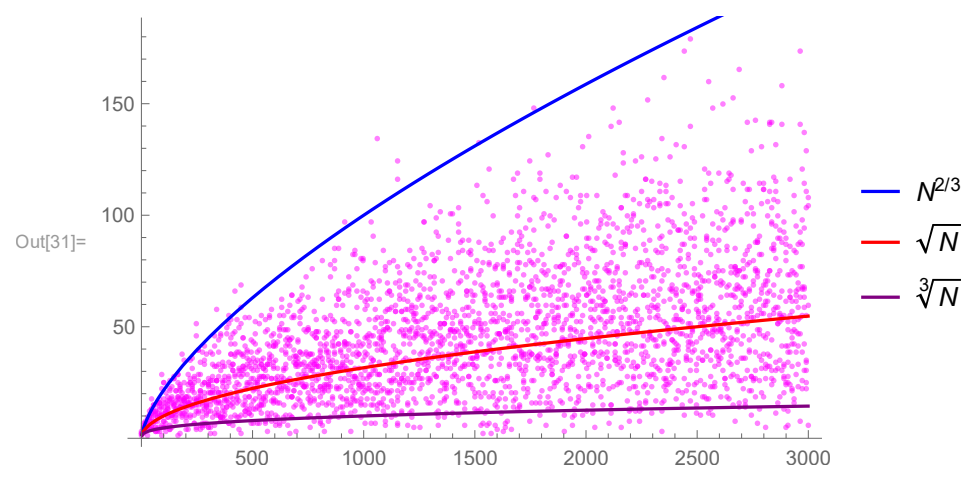

Figura 3: 3000 realizações para o tempo de absorção.

\section{Conclusões}

Foi mostrado que a proporção de vértices para o modelo Pula Morre converge para zero em probabilidade, usando a técnica planteada em Lebensztayn and Estrada 6]. Por sua vez, foram usadas técnicas de martingais, inspiradas no trabalho Sudbury [9], para mostrar que o tempo de absorção é $o_{p}(N+1)$.

\section{Agradecimentos}

Agradeço à Coordenação de Aperfeiçoamento de Pessoal de Nível Superior (Processo No. 88887.351976/2019-00 do Programa Nacional de Pós-Doutorado) e à Fundação de Amparo à 
Ciência e Tecnologia de Pernambuco-FACEPE (Processo No. BCT - 0217-1.02/19). Agradeço a meus supervisores Pablo Rodriguez e ao Alex Dias Ramos, pela supervisão e apoio. Agradeço também ao revisor anônimo pelas valiosas sugestões que ajudaram a melhorar o texto.

\section{A Distribuição de urnas vazias}

Nesta seção apresentaremos o clássico problema de ocupação, fazendo referência ao leitor interessado a Johnson [5, Seção 4 do Capítulo 10] para mais detalhes. Consideramos uma distribuição aleatória de $b$ bolas em $c$ caixas, de forma que são colocadas independentemente e uniformemente nas caixas. Seja $X$ a variável aleatória que denota o número de caixas vazias depois que as bolas tenham sido distribuídas. Então a função de massa de probabilidade de $X$ é dada por

$$
P(X=x)=\sum_{i=0}^{c-x}(-1)^{i}\left(\begin{array}{c}
x+i \\
i
\end{array}\right)\left(\begin{array}{c}
c \\
x+i
\end{array}\right)\left(1-\frac{x+i}{c}\right)^{b}, x=0,1, \ldots, c .
$$

Escrevemos $X \sim \operatorname{EmpBox}(b, c)$. Na sequência, usaremos as fórmulas para a esperança e a variância de $X$, que são dadas por

$$
\begin{aligned}
\mathbb{E}(X) & =c\left(\frac{c-1}{c}\right)^{b}, \mathrm{e} \\
\operatorname{Var}(X) & =c(c-1)\left(\frac{c-2}{c}\right)^{b}+c\left(\frac{c-1}{c}\right)^{b}-c^{2}\left(\frac{c-1}{c}\right)^{2 b} .
\end{aligned}
$$

\section{Referências}

[1] Nikki Cartern, Brittany Dygert, Matthew Junge, Stephen Lacina, Collin Litterell, Austin Stromme, and Andrew You. Frog model wakeup time on the complete graph, 2015.

[2] Benjamin Doerr and Marvin Künnemann. Tight analysis of randomized rumor spreading in complete graphs. In Proceedings of the Meeting on Analytic Algorithmics and Combinatorics, page 82-91, USA, 2014. Society for Industrial and Applied Mathematics.

[3] A.M. Frieze and G.R. Grimmett. The shortest-path problem for graphs with random arclengths. Discrete Applied Mathematics, 10(1):57 - 77, 1985. ISSN 0166-218X.

[4] Bernard Harris. Probability distributions related to random mappings. Ann. Math. Statist., 31(4):1045-1062, 12 1960. doi: 10.1214/aoms/1177705677.

[5] Johnson, N.; Kotz, S.; Kemp, A. Univariate discrete distributions. [S.l.]: John Wiley \& Sons, 1992. (Wiley series in probability and mathematical statistics: Probability and mathematical statistics).

[6] Elcio Lebensztayn and Mario Andrés Estrada. Laws of large numbers for the frog model on the complete graph. Journal of Mathematical Physics, 60(12):123302, 2019.

[7] Elcio Lebensztayn, Fábio Prates Machado, and Mauricio Zuluaga Martinez. Random walks systems with killing on Z. Stochastics, 80(5):451-457, 2008. doi: 10.1080/17442500701748609.

[8] Boris Pittel. On spreading a rumor. SIAM Journal on Applied Mathematics, 47(1):213-223, 1987. ISSN 00361399.

[9] Aidan Sudbury. The proportion of the population never hearing a rumour. Journal of Applied Probability, 22(2):443-446, 1985. doi: 10.2307/3213787. 